\title{
Factors associated with Pneumocystis carinii pneumonia in Wegener's granulomatosis $\dagger$
}

\author{
Bertrand Godeau, Jean-Luc Mainardi, Françoise Roudot-Thoraval, Eric Hachulla, \\ Loïc Guillevin, Le Thi Huong Du, Bernard Jarrousse, Philippe Remy, Annette Schaeffer, \\ Jean-Charles Piette
}

Service de Médecine Interne,

Hôpital Henri Mondor, Créteil, France

B Godeau

J-L Mainardi

A Schaeffer

Unité d'Évaluation

Clinique et

Biostatistique

F Roudot-Thoraval

Service de Néphrologie

P Remy

Service de Médecine

Interne,

Hôpital Claude

Huriez,

Lille, France

E Hachulla

Service de Médecine Interne,

Hôpital Avicenne,

Bobigny, France

L Guillevin

B Jarousse

Service de Médecine

Interne,

Hôpital La Pitié

Salpétrière,

Paris, France

L T Huong Du

J-C Piette

Correspondence to:

Dr Bertrand Godeau

Service de Médecine

Interne I,

Hôpital Henri Mondor 94010 Créteil, France.

Accepted for publication 31 August 1995

tThis work has been presented in part at the 1993 Annual Scientific Meeting of the American College of Rheumatology, San Antonio, Texas, USA. November 1993

\begin{abstract}
Objective-To determine the factors associated with the occurrence of Pneumocystis carinii pneumonia (PCP) in Wegener's granulomatosis (WG).

Methods-We retrospectively compared a group of 12 patients with WG and PCP (PCP group), with 32 WG patients without PCP followed over the same period in the same centres (control group).

Results-The mean delay of onset of PCP after the start of the immunosuppressive therapy was 127 (SD 128) days. Before treatment, the clinical and biological features of the two groups were similar, except for the mean lymphocyte count which was lower in the PCP group than in the control group $\left(1060 / \mathrm{mm}^{3} v 1426 / \mathrm{mm}^{3}\right.$; $\mathbf{p}=0.04)$. During treatment, both groups were lymphopenic. There was a significant difference between the lowest absolute lymphocyte count in each group $\left(244 / \mathrm{mm}^{3}\right.$ in the PCP group $v 738 / \mathrm{mm}^{3}$ in the control group; $p=0 \cdot 001$ ). During the first three months of treatment, the lymphocyte count was less than $600 / \mathrm{mm}^{3}$ at least once in 10 of the 12 patients in the PCP group and in 11 of the 32 patients in the control group $(p<0 \cdot 01)$. The mean cumulative dose of cyclophosphamide was greater in the PCP group than in the control group at the end of both the second $(1.55 \mathrm{mg} / \mathrm{kg} / \mathrm{day} v 0.99 \mathrm{mg} / \mathrm{kg} / \mathrm{day} ; \mathrm{p}=0.05)$ and the third $(1.67 \mathrm{mg} / \mathrm{kg} /$ day $v 0.97 \mathrm{mg} / \mathrm{kg} /$ day; $p=0.03$ ) months. However, in multivariate analysis, the only two factors independently and significantly associated with the occurrence of PCP were the pretreatment lymphocyte count $(p=0.018)$ and the lymphocyte count three months after the start of the immunosuppressive treatment $(p=0.014)$.
\end{abstract}

Conclusions-The severity of lymphocytopenia before and during immunosuppressive treatment is the factor best associated with PCP in WG.

(Ann Rheum Dis 1995; 54: 991-994)

The frequency of death from Wegener's granulomatosis (WG) has been substantially reduced by cyclophosphamide and glucocorticoid therapy. ${ }^{1}$ Despite its success, the treatment is frequently associated with side effects and severe infectious complications, ${ }^{2}$ among which Pneumocystis carinii pneumonia (PCP) is one of the most frequent and opportunistic, often resulting in death. ${ }^{3}$ It is therefore important to identify patients with WG who are at risk of PCP in order that they receive primary antipneumocystis prophylaxis. To determine the factors associated with the occurrence of PCP in WG, we compared retrospectively a group of 12 patients with both WG and PCP, with all patients suffering from WG, but without PCP, followed in the same units over the same period.

Patients and methods

The study was carried out in four medical centres that contributed six, 12, 12, and 14 patients.

\section{INFORMATION COLLECTED}

Twelve cases of PCP among patients suffering from WG followed in these four centres between 1984 and 1992 (PCP group) were compared retrospectively by three investigators; a control group of 32 comprised all consecutive patients suffering from WG without PCP followed regularly over the same period in the same centres. The PCP and control groups were compared for usual clinical and biological data collected on diagnosis and during the follow up period. The doses and mode of administration (by mouth, or intravenous pulses) of corticosteroids and cytotoxic drugs received before and after the date of diagnosis were recorded. The doses of corticosteroids are expressed as prednisone equivalent doses.

All patients included fulfilled the diagnostic criteria for WG proposed by the American College of Rheumatology. ${ }^{4}$ Patients were excluded from the study if they were infected with human immunodeficiency virus (HIV), they suffered from associated neoplasic diseases, or they received primary anti-PCP prophylaxis.

STATISTICAL ANALYSIS

Data collected before diagnosis and during the follow up period were compared between PCP and control groups using the $\chi^{2}$ test and Fisher's exact test, if necessary, for categorical variables, and the non-parametric MannWhitney test for quantitative variables. Nonparametric regression analysis (Spearman's rank correlation) was used to test the 
relationship between pairs of quantitative parameters. Multivariate analysis was performed using stepwise logistic regression analysis (BMDP Statistical Software, 3SLR). Two tailed $p$ values of 0.05 or less were considered significant.

\section{Results}

PATIENT CHARACTERISTICS

PCP group-Some of the clinical and biological characteristics of the 12 patients of the PCP group have been described in a previous study. ${ }^{5}$ Briefly, the mean delay before onset of PCP was 128 (SD 127) days after the beginning of immunosuppressive therapy (median 90 days, range 50-510). PCP developed in the three months following the start of the treatment in most patients (eight of 12 patients) and was not chronologically related to the tapering of corticosteroid treatment.

Control group-Among the 51 patients suffering from WG without PCP followed in the same centres over the same period, 32 were included in the control group. Nine patients were not included for the following reasons: localised WG without systemic manifestations (three), HIV infection (one), and primary antiPCP prophylaxis (five). Ten other patients were excluded because insufficient data were available or they were not followed regularly in the centres involved in the study. The mean period of observation of the control group was $19.5(9.5)$ months from the date of diagnosis of WG.

\section{COMPARISON AT THE TIME OF DIAGNOSIS OF WG}

The PCP and control groups had similar initial clinical presentations (table). The proportions of patients who had received corticosteroid treatment before WG diagnosis were similar in the two groups, and no patient in either group received cytotoxic agents before diagnosis of WG. The only statistically significant difference between the two groups was the mean value of the lymphocyte count, which was

Patients' characteristics on diagnosis of Wegener's granulomatosis (WG)

\begin{tabular}{|c|c|c|c|}
\hline & $\begin{array}{l}P C P \text { group } \\
(n=12)\end{array}$ & $\begin{array}{l}\text { Control group } \\
(n=32)\end{array}$ & $p \Phi$ \\
\hline $\begin{array}{l}\text { Mean age }(y r) \\
\text { Sex ratio }(F: M)\end{array}$ & $\begin{array}{c}51(13) \\
4 \cdot 8\end{array}$ & $49(11)$ & 0.4 \\
\hline Sex ratio $(\bar{F}: M)$ & $4: 8$ & $7: 25$ & $0 \cdot 7$ \\
\hline $\begin{array}{l}\text { Mean interval between first } \\
\text { clinical manifestations and } \\
\text { diagnosis (months) }\end{array}$ & $17(8)$ & $13(26)$ & 0.6 \\
\hline \multicolumn{4}{|l|}{ Frequencies of involvement $†$} \\
\hline Upper airway & $11(92)$ & $31(97)$ & \\
\hline Lung & $9(75)$ & $26(81)$ & $0 \cdot 3$ \\
\hline Kidney & $9(75)$ & $27(84)$ & \\
\hline $\begin{array}{l}\text { Corticosteroid treatment } \\
\text { before diagnosis of } \mathrm{WG} \dagger^{\star}\end{array}$ & $2(16)$ & $10(31)$ & $0 \cdot 6$ \\
\hline Neutrophil leucocyte count $\left(/ \mathrm{mm}^{3}\right)$ & $9800(4100)$ & $10200(4800)$ & 0.8 \\
\hline LDH (x normal value) & $1 \cdot 1(0 \cdot 2)$ & $1 \cdot 1(0 \cdot 2)$ & 0.9 \\
\hline Serum albumin concn $(g / 1)$ & $31(5)$ & $31(6)$ & $0 \cdot 8$ \\
\hline Gammaglobulin $(g / 1)$ & $10(3)$ & $13(6)$ & 0.3 \\
\hline Creatinine concn $(\mu \mathrm{mol} / \mathrm{l})$ & $351(337)$ & $238(228)$ & $0 \cdot 2$ \\
\hline Lymphocyte count $\left(/ \mathrm{mm}^{3}\right)$ & $1060(670)$ & $1426(509)$ & 0.04 \\
\hline
\end{tabular}

Values are mean (SD) or tnumber (\%).

\$Pneumocystis carinii pneumonia (PCP) and control groups compared using the $\chi^{2}$ test and Fisher's exact test for categorical variables, and the non-parametric Mann-Whitney test for quantitative variables. Lactate dehydrogenase (LDH) and creatinine values are maximum values observed in the month preceeding diagnosis of Wegener's granulomatosis; values for serum $\star$ albumin, gammaglobulin, neutrophil and lymphocyte counts a lower in the PCP group: 1060 (670)/ $\mathrm{mm}^{3}$ (median $800 / \mathrm{mm}^{3}$, range 140-2600) $v$ $1426(509) / \mathrm{mm}^{3}$ (median $1220 / \mathrm{mm}^{3}$, range 576-2520) $(\mathrm{p}=0.04)$.

\section{COMPARISON AFTER THE START OF THE} TREATMENT

Among the extreme values observed during the study, the only parameter which was significantly different between the two groups was the lymphocyte count: both groups were lymphocytopenic, but the smallest lymphocyte count was significantly less in the PCP group (244 (362) $/ \mathrm{mm}^{3}$ (range 0-1200) v 738 (543) $/ \mathrm{mm}^{3}$ (range $60-2400$ ); $\mathrm{p}=0.001$ ). As PCP occurred mainly in the three months after the start of the treatment, we compared the lymphocyte counts during the first, second, and third months of treatment. The minimum lymphocyte counts during each of these periods were significantly lower in the PCP group than in the control group (fig 1). However, the mean decreases in the minimum lymphocyte count between the start of the treatment and the second month did not differ significantly between the groups $(-76$ lymphocytes $/ \mathrm{mm}^{3}$ (median 0) in the PCP group compared with +35 (median +34 ) in the control group; $p=0 \cdot 2$ ). In non-parametric regression analysis, the minimum lymphocyte counts during the first and the second months of treatment correlated significantly with the pretreatment lymphocyte count $(r=0.47$, $\mathrm{p}<0.01$ and $r=0.36, \mathrm{p}<0.05$, respectively). During the first three months after the start of treatment, the value of the lymphocyte count that best discriminated between PCP and control groups was $600 / \mathrm{mm}^{3}$ : the lymphocyte count was less than this value at least once in 10 of the 12 patients in the PCP group, but in only 11 of the 32 patients in the control group $(\mathrm{p}<0.01)$. The CD4 and CD8 lymphocyte subsets were not studied because the required data were rarely available.

Creatinine values during treatment showed a tendency to be greater in the PCP group (442 (361) $\mathrm{mmol} / 1$ v 254 (222) $\mathrm{mmol} / \mathrm{l})$, but this difference did not reach significance $(p=0.06)$. The extreme values of other data collected during the period of the study (maximum values for lactate dehydrogenase concentrations and minimum values for neutrophil counts and serum albumin and gammaglobulin concentrations) were similar in the two groups (data not shown).

\section{COMPARISON OF IMMUNOSUPPRESSIVE} TREATMENTS

All patients of both groups received daily corticosteroids and cyclophosphamide, but the doses and routes of administration of these drugs were not standardised among the various teams participating in the study. The most recent patients received cyclophosphamide via intermittent IV pulses rather than daily and orally, to minimise cumulative toxicity; ${ }^{6}$ the proportion of patients treated by intermittent pulses was not statistically different between 


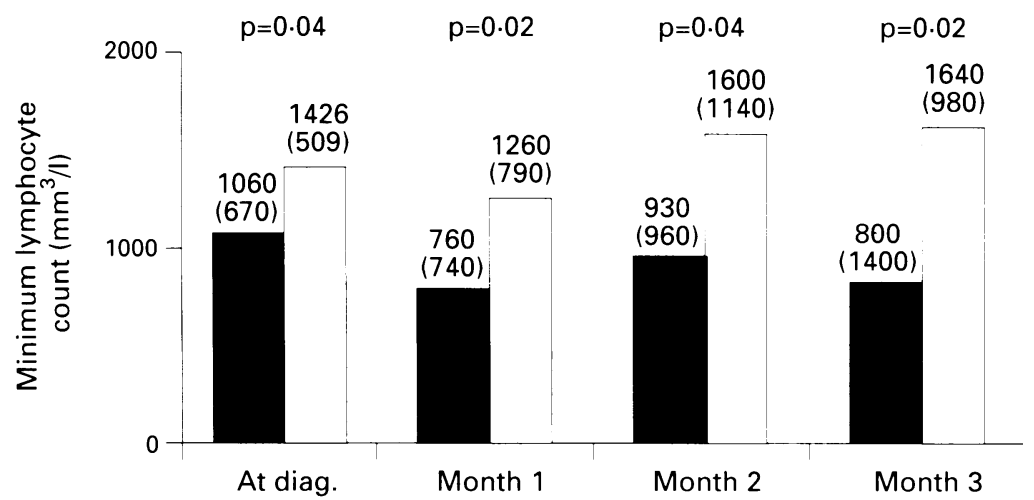

Figure 1 Mean (SD) minimum lymphocyte counts in the Pneumocystis carinii pneumonia (PCP) group ( $\square$ ) and control group ( $\square$ ) during the first, second, and third months after the start of treatment. Values of $p$ are for comparisons between groups (non-parametric Mann-Whitney test). count at month 3 at a cut off of $600 / \mathrm{mm}^{3}$ $(p=0.014)$.

\section{Discussion}

As reported by Hoffmann et al, ${ }^{3}$ PCP appears to be a frequent event in cases of WG, though we could not accurately estimate the frequency from this retrospective study. It is important, therefore, to determine factors associated with PCP in order that primary anti-PCP prophylaxis may be given to patients with WG. Our results indicate that lymphopenia is the most important factor associated with PCP in WG.

Lymphocytopenia is known to contribute to the development of PCP in patients with various underlying diseases including AIDS and malignancies, and systemic lupus erythematosus (SLE) patients receiving cytotoxic treatment. $^{--9}$ We report similar findings for patients with WG: the lymphocyte count was significantly decreased during the first three months of treatment in patients who developed PCP. Surprisingly, the lymphocyte count before treatment was also significantly decreased in the PCP group, and was an independent factor associated with PCP in multivariate analysis. All other data collected at the time of diagnosis of WG were similar in the two groups. Pretreatment lymphocytopenia may thus be a marker of active WG with a more impaired immune response, and the standard lymphocyte count may be useful for predicting the risk of PCP: a threshold of 600 lymphocytes per $\mathrm{mm}^{3}$ during the first three months after diagnosis of WG defined the group of patients at greater risk of PCP, because the lymphocyte count almost invariably decreased below this value at least once in the PCP group. It is unclear whether evaluation of peripheral lymphocyte subset distribution may better define the risk of PCP; it has been demonstrated that the immunophenotypic distribution of lymphocytes is normal in WG, ${ }^{6}{ }^{10}$ but there is a conflicting report. ${ }^{11}$

All of our patients received high dose corticosteroids, and the importance of steroids as a risk factor for PCP has been emphasised in other immunosuppressive conditions. ${ }^{12}$ Unlike patients with SLE who develop PCP, ${ }^{9}$ our WG patients with PCP received doses similar to those given to the control group. However, these retrospective data are insufficient to affirm that corticosteroids were not relevant to the incidence of PCP, because the doses administered were high in both groups. In contrast, the PCP group received a significantly greater cumulative dose of cyclophosphamide. It has not previously been determined if PCP in patients with $\mathrm{WG}$ is attributable to corticosteroid treatment alone, or whether associated immunosuppressive treatment is also involved. ${ }^{3}$ Our study suggests that the dose of cyclophosphamide is important. However, we cannot affirm from this retrospective study that the greater dose of cyclophosphamide administered to the PCP group does not simply reflect a more aggressive management for more severe disease. The 
results of the multivariate analysis showed that lymphocyte count before and during treatment appears to be better than cyclophosphamide as a predictive factor for risk of PCP.

Primary anti-PCP prophylaxis is effective in AIDS patients. ${ }^{13}$ The frequency and severity of PCP in WG justifies a similar prophylaxis, mainly with trimethoprim-sulphomethoxazole, as it has been suggested that this drug may also be useful in the treatment of WG. ${ }^{14}$ However, in view of the potential side effects associated with this drug, particularly cutaneous and haematological toxicity, our results suggest that primary prophylaxis is indicated most appropriately for patients whose lymphocyte count decreases to less than $600 / \mathrm{mm}^{3}$ during the first three months of treatment. Further studies are required to define these limits more clearly.

We wish to thank Dr I Theodorou for her expert revision of this manuscript, Drs M Bagot, D Bataille, M Denis, P Y Hatron, C Jacquot, P Leclerc, J C Roujeau, G Rostoker, and D Valeyre for their co-operation with patient recruitment, and A Edelman for their co-operation with

1 Hoffman G S, Kerr G S, Leavitt R Y, et al. Wegener's granulomatosis: an analysis of 158 patients. Ann Intern Med 1992; 116: 488-98.

2 Bradley J D, Brandt D K, Katz B P. Infectious complication of cyclophosphamide treatment of vasculitis. Arthritis Rheum 1989; 32: 45-53.

3 Hoffman G S, Leavitt R Y, Kerr G S, Fauci A. The treatment of Wegener's granulomatosis with glucocorti- coids and methotrexate. Arthritis Rheum 1992; 35: 1322-9.

4 Leavitt R Y, Fauci A S, Bloch D A, et al. The American College of Rheumatology 1990 criteria for the classification of Wegener's granulomatosis. Arthritis Rheum 1990; 33: 1101-7.

5 Godeau B, Coutant-Perronne V, Le Thi Huong Du, et al. Pneumocystis carinii in the course of connective tissue disease: report of 34 cases. $f$ Rheumatol 1994; 21 : 246-51.

6 Hoffman G S, Leavitt R Y, Fleischer T A, Minor J R, Fauci A S. Treatment of Wegener's granulomatosis with intermittent high dose intravenous cyclophosphamide. Am F Med 1990; 89: 403-10

7 Kovacs J A, Hiemenz J W, Macher A M, et al. Pneumocystis carinii pneumonia: a comparison between patients with acquired immunodeficiency syndrome and patients with other immunodeficiencies. Ann Intern Med 1984; 100: 663-71.

8 Liam C K, Wang F. Pneumocystis carinii pneumonia in patients with systemic lupus erythematosus. Lupus 1992; 1: 379-85.

9 Porges A J, Beattie S L, Ritchlin C, Kimberly R P, Christian C L. Patients with systemic lupus erythematosus at risk for pneumocystis carinii pneumonia. $\exists$ Rheumatol 1992; 19: 1191-4.

10 Rasmussen N, Peterson J, Ralfkiaer E, et al. Spontaneous and induced immunoglobulin synthesis and antineutrophil cytoplasmic antibodies in Wegener's granulomatosis: relation to leukocyte subpopulations in blood and active lesions. Rheumatol Int 1988; 8: 153-8.

11 Jarrousse B, Guillevin L, Bindi P, et al. Increased risk of Pneumocystis carinii pneumonia in patients with Wegener's granulomatosis. Clin Exp Rheumatol 1993; 11: 615-21.

12 Ruskin J. Parasitic diseases in the compromised host. In: Rubin T H, Young L S, eds. Clinical approach to infection in the compromised host, 2nd edn. New York, London: Plenum Medical Book Company, 1987; 253-75.

13 Kovaks J A, Masur H. Pneumocystis carinii pneumonia; therapy and prophylaxis. $\mathcal{Y}$ Infect Dis 1988; 158: 254-9.

14 De Remee R A. The treatment of Wegener's granulomatosis with trimethoprim/sulfamethoxazole: illusion or vision? Arthritis Rheum 1988; 31: 1068-72. 